\title{
Peertechz
}

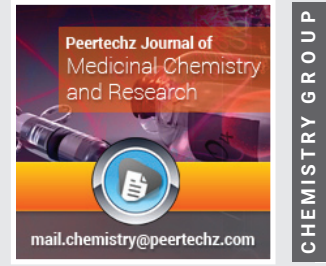

\section{Bioactive Compounds and}

\section{Biological Assays of different}

\section{Prosopis Juliflora Extracts} against Groundnut Aphid

\section{Mulate Zerihun ${ }^{1 *}$ and Estifanos Ele ${ }^{2}$}

${ }^{1}$ Agricultural chemistry, Melkassa Agriculture Research Center, EIAR, P. O. Box 2003, Addis Ababa, Ethiopia

${ }^{2}$ Department of Chemistry, College of Natural Sciences, Addis Ababa University, P. O. Box 1176, Addis Ababa, Ethiopia
Received: 31 October, 2020

Accepted: 12 November, 2020

Published: 17 November, 2020

*Corresponding author: Mulate Zerihun, Agricultural chemistry, Melkassa Agriculture Research Center, EIAR, P. O. Box 2003, Addis Ababa, Ethiopia, Tel: +251116461086; E-mail:mulatezerihun@yahoo.com ORCID: https://orcid.org/0000-0002-1068-0295 Keywords: Prosopis juliflora; Bioactive compound; Plant extraction; Isolation; Insecticidal properties; Natural products

https://www.peertechz.com

\section{Check for updates}

\section{Abstract}

Natural products from medicinal plants, either as pure compounds or as standardized extracts, provide unlimited opportunities for different purposes. Botanical preparations of medicinal plants for insecticidal usage contain various types of bioactive compounds. The aim of this paper focuses on the botanical evaluation of stem bark, seed and leaf extracts of Prosopis juliflora for their insecticidal effect against groundnut (Aphis craccivora) aphids. Extracts with the concentrations of 1 , $2.5,5,10$ and $15 \%$ weight by weight percentage were tested. The highest mortality rate of the groundnut aphid that was recorded after $12 \mathrm{~h}$, due to the methanolic and dichloromethane extracts, was $83.33 \%$. The advantages and problems of different extraction techniques were tested and discussed. Different chromatographic techniques were employed to identify, isolate and characterize secondary metabolites. Two compounds, 4-(2-aminoethyl) phenol (1) and 3-0-methyl-chiro-inositol (2) were isolated from the methanolic extract of the leaves part. Structural elucidations of the isolated compounds were based on their ${ }^{1} \mathrm{H}$ NMR, ${ }^{13} \mathrm{C}$ NMR, UV and IR spectra.

\section{Introduction}

The high diversity of plants is an available source of useful compounds. People extract plants and use them for different purposes. Ancient habitats found plant extracts as efficient medicines for the relief of pains or alleviation of the symptoms of the disease, as poisons for use in warfare and hunting, as effective agents for euthanasia and capital punishment. They were also used as narcotics, hallucinogens, or stimulants to relieve the tedium, or alleviate fatigue and hunger. Many of these natural products are still used today and usually for the same general purposes [1].

Natural products are organic compounds of natural origin that are unique to one organism or common to a small number of closely related organisms. The use of natural products as medicines, poisons, hallucinogens, stimulants, perfumes, flavoring agents, insecticides, insect antifeedants, fungicides, plant growth-regulating hormones, molluscicides, etc., is well known. It is therefore not difficult to understand what motivates chemists to isolate and characterize natural products. The characterization of new compounds is usually followed by their synthesis and the study of their biological activity and biosynthesis. One of the most interesting phenomena that emerged in the last few years is the realization that natural products that have been considered useless do have functions in the organisms from which they originate. It is recognized that many of them have vital roles as mediators of ecological interactions, thereby ensuring the continued survival of a particular organism. Despite the vast number and structural diversity of metabolites, almost all arise by one of three biosynthetic pathways or by a combination of two or more of these pathways. These are known as the acetate, mevalonate and shikimate pathways [2].

Prosopis belongs to the family Leguminosae (Fabaceae), subfamily Mimosoideae. Of the 44 species of Prosopis, 40 
are native to the Americas, three to Asia and one to Africa. In the Americas, Argentina has 28 native species, of which 13 are endemic [3]. Prosopis species grow in a wide array of environments and are commonly not restricted by soil type, $\mathrm{pH}$ or salinity. They grow in semi-arid and arid tracts of tropical and sub-tropical regions of the world and are spreading fast because the leaves are unpalatable and animals do not digest its seeds [4].

P.juliflora is a shrub or small tree, a kind of mesquite. Within Prosopis species, there are trees and shrubs of varying size, mainly characterized by the presence of thorns and prickles. The term "mesquite" includes all leguminous trees of the genus Prosopis, of which nine species are found in the highly arid environments of Mexico. These species are highly recognized for their properties as windbreakers, soil binders, and sand stabilizers, as well as their ability to grow in the poorest soils and to survive in areas where other trees cannot survive [5]. P.juliflora was introduced in Ethiopia as a biological soil and water conservation agent during the late 1970s. Now it is becoming a major threat because of its invasive nature. Some reports indicate that P. juliflora is widely distributed in Ethiopia [6,7].

Leaves and extracts of the leaves of Prosopisspecies have been noted to have bio-control properties. Extracts of P. juliflora are effective against some weed species, insects, nematodes, pathogenic fungi and viruses. Leaf extracts from P. juliflora have been found to inhibit germination in a number of species ) [8]. The uses of P. juliflora leaves to control the invasive weed Parthenium hysterophorus has been assessed, with a noted reduction in seed germination [9].

The numerous goods and services obtained from P. juliflora have led to global introductions of the species. In South Africa, pods are collected to produce organic medicines ('manna') that are said to have properties that stabilize blood sugar level in humans [10]. Products from this plant have also been used for human consumption in bread, biscuits, sweeties, syrup and liquors [11]. Extracts of P.juliflora seeds and leaves have shown several in vitro pharmacological effects such as antibacterial, antifungal, and anti-inflammatory properties [12]. The aqueous extracts of P. juliflora have also been known for its antibacterial activity against different phytopathogenic bacteria [13]. A water-soluble mixture of alkaloids from P. juliflora leaves was found to be more active against gram-positive bacteria than commercial antibiotics such as bacitracin, chloromycetin, gentamicin or trimethoprim [14]. Therefore, the objective of this study was to investigate the phytochemical composition of P.juliflora and insecticidal activities of its extracts against groundnut aphid.

\section{Materials and methods}

\section{General}

All solvents, standards and reagents used were of the analytical grade, that purchased from Fisher Scientific UK, Bishop Meadow Road Loughborough, Leics, LEll 5RG, UK, except for fatty acid standards that were purchased from
Sigma-Aldrich Chemie GmbH, Riedstrasse2, D-89555 Steinheim Germany.

\section{Instruments}

GCMS analysis was carried out with Agilent technologies 7820A GC and 5977E MSD system equipped with an autosampler. Chromatographic separations were carried out using column length $30 \mathrm{~m} \times 0.25 \mathrm{~mm}$ and column phase thickness $0.25 \mu \mathrm{m}$ of $\mathrm{HP}-88$ coated with $100 \%$ poly (dimethylsiloxane). Injection mode was splitless and $1 \mu \mathrm{l}$ injection volume in total run time 31.31 minutes under helium carrier gas was injected. The fatty acid methyl esters were run on GCMS and their results were expressed as mean values \pm standard deviation ( $\mathrm{M} \pm \mathrm{STD}$ ).

NMR spectra were recorded on Bruker Avance $400 \mathrm{MHz}$ spectrometers. For ${ }^{1} \mathrm{H}$ NMR (400 MHz) and ${ }^{13} \mathrm{C}$ NMR (100.6 $\mathrm{MHz})$ spectra, the chemical shifts $(\delta)$ are reported in parts per million (ppm) relative to TMS. The $\delta$ values are referenced to $\mathrm{CHCl}_{3}$ in $\mathrm{CDCl}_{3}$ at $7.27 \mathrm{ppm}$.

\section{Chromatography}

Analytical TLC was run on MerckKieselgel 60 F254. Plates were visualized under UV light and by spraying with Vanillin: $5 \%$ $\mathrm{H}_{2} \mathrm{SO}_{4}$ in $\mathrm{MeOH}$ followed by heating for a few seconds.

\section{Column chromatography}

Silica gel 60 (Merck), particle size 0.063-0.200 (70-230 mesh ASTM) was used for column chromatography.

\section{Plant material}

The stem bark, seeds and leaves of P. juliflora were collected from Amibara Woreda (Afar region) 9० 60' 45" N latitude and $40^{\circ} 9^{\prime} 32^{\prime \prime}$ E longitude and at an altitude of 740 meters above sea level), $280 \mathrm{~km}$ northeast of Addis Ababa, during October 2016. The plant specimen was identified at the Biology Department, AAU. The samples were collected in sterile polyethylene bags. The fresh samples were transported in icebox and were preserved in a deep freezer until processing.

\section{Plant materials preparation}

The stem bark of P. juliflora was chopped into small pieces and dried at room temperature for two weeks. The dried seeds and stem bark were milled using a "knife" mill. The fresh leaves were frozen in liquid nitrogen and crushed with a mortar and pestle.

\section{Extraction}

The powdered plant materials were extracted by hydrodistillation, Soxhlet, and solvent extractions. The crude extracts were evaluated for the insecticidal activity against groundnut aphids under laboratory conditions with different concentrations. Based on the preliminary insecticidal evaluation phytochemical study was conducted on methanol leaf extract of P. juliflora as follows.

The fresh leaf of P. juliflora was cut into small pieces, frozen in liquid nitrogen and ground. The ground leaf $(5 \mathrm{~kg})$ 
was soaked in $\mathrm{MeOH}(8 \mathrm{~L})$ for $72 \mathrm{~h}$ at room temperature with occasional stirring and shaking. The extracts were filtered first through a fresh cotton plug and then with a Whatman No. 1 filter paper. The solvent was removed by a rotary evaporator at $35{ }^{\circ} \mathrm{C}$ to afford a dark green crude extract (309 g). Solventsolvent partition (Figure 1) was done by using the protocol developed by Kupchan and Tsou [15] and modified by Wagenen, et al. [16]. Thus, the crude extract was dissolved in $\mathrm{MeOH}$ $(400 \mathrm{~mL})$ and defatted by soaking in $\mathrm{n}$-hexane $(0.8 \mathrm{~L})$ for 48 $\mathrm{h}$ with occasional stirring and shaking. The hexane extract was subjected to a rotary evaporator to afford $10.7 \mathrm{~g}$ of hexane extract. The residue obtained after removal of $\mathrm{MeOH}$ was washed with DCM (0.8L) and removal of the solvent afforded $8.9 \mathrm{~g}$ of the DCM-soluble material. The final residue was dried to afford $289 \mathrm{~g}$ of hexane and DCM-insoluble material. Figure 1 summarizes the extraction procedure.

\section{Isolation of compounds}

The residue (FR 3, 30g) was applied on a silica gel column and eluted with DCM-MeOH mixtures of increasing polarity. A total of 49 fractions (each $100 \mathrm{~mL}$ ) were collected as shown in
Table 1. The progress of separation was monitored by analytical TLC with DCM-MeOH solvent system and fractions of similar TLC profiles were combined to give twelve fractions (Table 1).

Fraction 6' was subjected to further purification over a short silica gel column with EtOAc: $\mathrm{MeOH}$ mixtures in increasing polarity. A total of 13 fractions (each $10 \mathrm{~mL}$ ) were collected. Fractions 1 to 5 were collected using EtOAc-methanol (9:1) solvent system. Fraction 6 eluted with EtOAc:MeOH (8:2) afforded white crystals (31 $\mathrm{mg}$ ) and the obtained crystal was identified through different physical and spectroscopic techniques (UV, NMR, and IR) to be 4-(2-aminoethyl) phenol (1).

Fraction 9' was concentrated using a rotary evaporator to afford a residue $(989 \mathrm{mg})$. The obtained fraction residue was applied on a silica gel column and eluted with $\mathrm{CHCl}_{3}: \mathrm{MeOH}$ mixtures in increasing polarity. A total of 28 fractions (each 25 $\mathrm{mL}$ ) were collected. Fraction 13 , which was eluted with $\mathrm{CHCl}_{3}$ : $\mathrm{MeOH}$ (7.5:2.5) afforded a white crystalline solid (79mg). The obtained white crystals were identified through different physical and spectroscopy techniques (UV, NMR, and IR) to be 3-O-methyl-chiro-inositol (2).

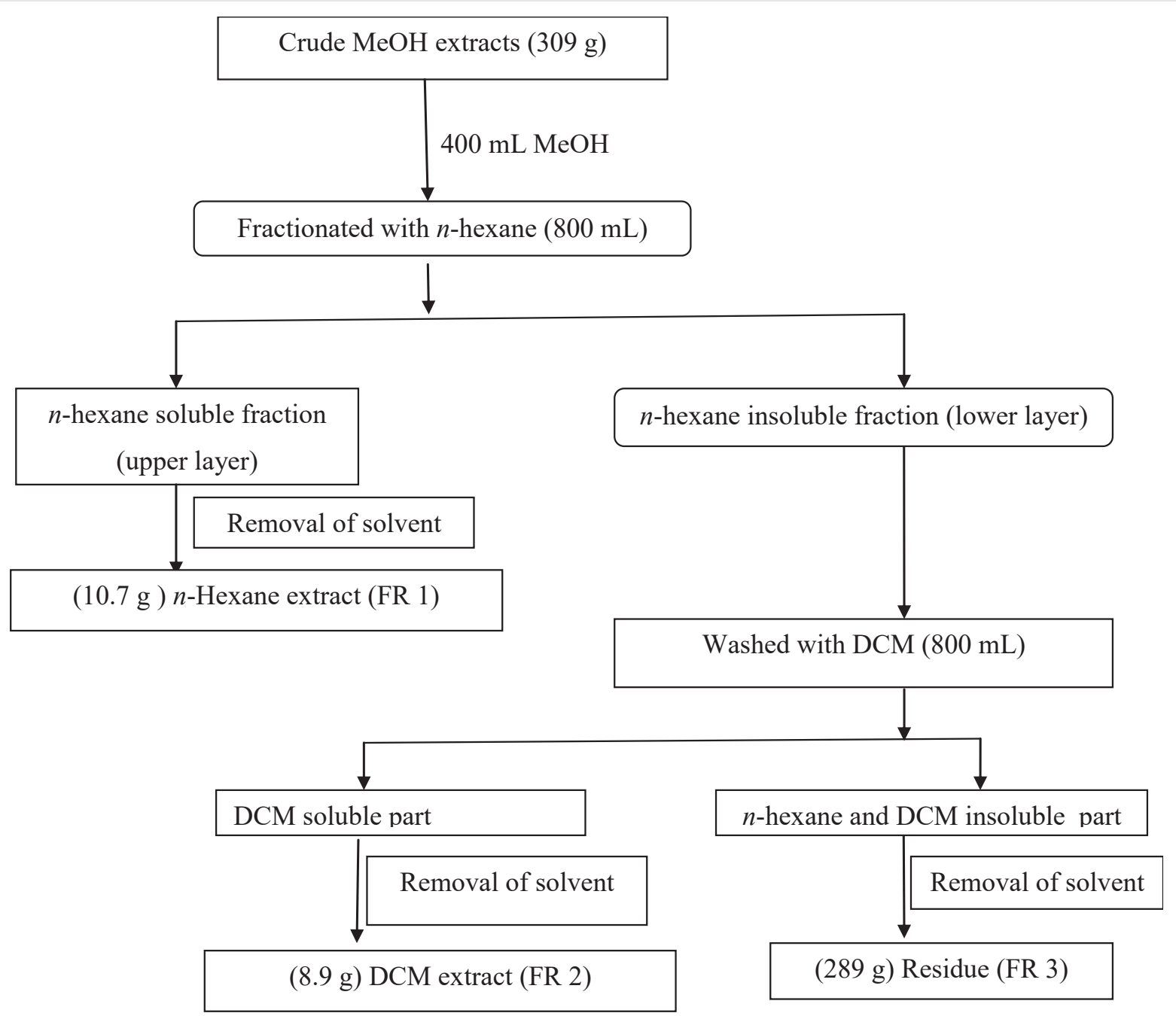

Citation: Zerihun M, Ele E (2020) Bioactive Compounds and Biological Assays of different Prosopis Juliflora Extracts against Groundnut Aphid. Open Journal of Chemistry 6(1): 021-029. DOI: https://dx.doi.org/10.17352/ojc.000020 
Table 1: Fractionations of the $\mathrm{MeOH}$ extract and weights of combined fractions.

\begin{tabular}{|c|c|c|c|c|c|c|}
\hline $\begin{array}{l}\text { Solvent } \\
\text { system }\end{array}$ & Ratio & Fractions & $\begin{array}{c}\text { Volume } \\
\text { (mL) }\end{array}$ & $\begin{array}{l}\text { Fractions } \\
\text { combined }\end{array}$ & Code & $\begin{array}{c}\text { Weight } \\
\text { (mg) }\end{array}$ \\
\hline DCM-MeOH & 10.0: 0 & $1 \& 2$ & 200 & $1 \& 2$ & $1^{\prime}$ & 29 \\
\hline$"$ & $9.5: 0.5$ & $3 \& 4$ & 200 & 3 & $2^{\prime}$ & 107 \\
\hline$“$ & $9.0: 1$ & $5-10$ & 600 & $4-6$ & $3^{\prime}$ & 307 \\
\hline$"$ & $8.5: 1.5$ & $11-15$ & 500 & $7-10$ & $4^{\prime}$ & 141 \\
\hline$"$ & 8.0:2.0 & $16-21$ & 600 & $11-13$ & $5^{\prime}$ & 826 \\
\hline$"$ & $7.5: 2.5$ & $22-26$ & 500 & $14-16$ & $6^{\prime}$ & 234 \\
\hline$"$ & $7.0: 3.0$ & $27-34$ & 800 & $17 \& 18$ & $7^{\prime}$ & 241 \\
\hline$"$ & $6.5: 3.5$ & 35-39 & 500 & $19-21$ & $8^{\prime}$ & 203 \\
\hline$"$ & $6.0: 4.0$ & $40-47$ & 800 & $22-26$ & $9^{\prime}$ & 989 \\
\hline$"$ & $5.0: 5.0$ & 48 & 100 & $27-34$ & $10^{\prime}$ & 2070 \\
\hline$"$ & $0: 10.0$ & 49 & 100 & $35-41$ & $11^{\prime}$ & 1034 \\
\hline- & - & - & - & $42-49$ & $12^{\prime}$ & 794 \\
\hline
\end{tabular}

\section{Identification of compounds}

The structures of isolated compounds were elucidated using physical parameters and spectroscopic techniques. Detailed analysis is given in the results and discussion sections.

\section{Bioassays}

The insecticidal activities of stem bark, seed and leaves extracts of P. juliflora were evaluated using dosage-dependent bioassays. Solutions of test materials were prepared by dissolving extracts in dimethyl sulfoxide by a method prescribed by Musabyimana et al.,. Five different concentrations were obtained by dissolving samples in $10 \%$ DMSO-water solutions to get emulsions containing 1.0, 2.5, 5.0, 10.0 and $15.0 \mathrm{w} / \mathrm{w} \%$. Equivalent quantities of DMSO-water solutions were used as solvent controls. Equivalent quantities of deionized water and blank controls were used as positive and negative controls respectively. Stem bark and leaves essential oil extracts were used in these bioassays. In the case of essential oil, an equivalent amount of deionized water and saturated sodium chloride were used as positive and solvent controls respectively. In each treatment three replication, each containing 10 aphids were used. Bioassays were carried out at room temperature (28 $\pm 2{ }^{\circ} \mathrm{C}$ ) in $30 \mathrm{~cm}$ diameter petri dish plates containing circular $33 \mathrm{~mm}^{2}$ Whatman \#1 filter paper inside each well. 1mL sample solution was added to the upper part of the petri dish. After the application of the solutions, aphids were maintained at controlled temperature $\left(28 \pm 2{ }^{\circ} \mathrm{C}\right)$ and humidity $(70 \% \pm 10 \%)$. Mortality rates were recorded after 12, 24, 48, 72 and 96 hrs after applying the solutions. Following the same procedure, dichloromethane and methanol extracts of seed, stem bark and leaves of P. juliflora were evaluated for their insecticidal activities.

\section{Results and discussion}

\section{Bioassay}

It is known that plants synthesize structurally diverse and complex secondary metabolites for different purposes such as defense, communication (signaling), etc. Secondary metabolites isolated from plants have attracted much attention because of their unique biological activities. Many of them are believed to act as pheromones, antifeedants or repellents, and as growth regulators [17]. The present work has investigated the effects of extracts of P. juliflora against groundnut aphid. The results obtained from this study are discussed below.

\section{Bioassays of extracts of P. juliflora against groundnut aphid}

The insecticidal activities of extracts of P. juliflora that were obtained through hydro distillation, solvent extraction and Soxhlet extraction were evaluated against groundnut aphid (Aphis craccivora). Insect mortality was evaluated using dosage-dependent bioassays as described in the experimental section and the results were recorded after 12, 24, 48, 72 and $96 \mathrm{~h}$. All measurements were done in triplicate analysis and the results were recorded as mean \pm standard deviation (SD). Statistical analyses were indicated that all of the extracts showed a dependence between mortality and concentration $(\mathrm{P}<0.0001)$ (Table 2$)$. The calculated mean mortalities of groundnut aphids are presented in Table 2.

The results presented in Table 2 summarize the bioassays of the extracts of P. juliflora that have promoted statistically significant mortality using Duncan's significance test [18] for at least one concentration compared with the control. Insecticidal activities higher than $50 \%$ at any tested concentrations were considered to show significant differences. The highest mortality for groundnut aphid was recorded at $1 \%$ concentration in $24 \mathrm{~h}$. At this concentration, the groundnut aphids encountered total death. The recorded insecticidal activities of the plant extracts showed statistical significance $(P<0.0001)$ for each concentration when compared to the controls. From Table 2, the plant extracts and concentrations were shown a significant difference in aphid mortality. Highly significant differences were observed between different extracts and different concentrations. This means that aphid mortality is affected by differences in plant extracts, concentration, and types of extracts used.

Figure 2 shows the mean mortality rates of groundnut aphids at the lowest concentration. Among the extracts of the leaves and stem bark, the methanol extracts were caused higher death when compared to other extracts. On the other hand, among the extracts of the seeds, the DCM extract has killed the aphids to a greater extent compared to other extracts. Besides, the highest mortality was recorded due to the leaf $\mathrm{MeOH}$ extracts compared to other plant parts. As shown in Figure 2, at the lowest concentration (1\%) the $\mathrm{MeOH}$ extract of the leaves of P. juliflora showed the highest insecticidal activity after $12 \mathrm{~h}$ of the treatment.

The $\mathrm{LC}_{50}, \mathrm{LC}_{95}, \mathrm{LT}_{50}$ and $\mathrm{LT}_{95}$ values were obtained through probit analysis ) [19]. The $\mathrm{LC}_{50}$ values were showed that $\mathrm{MeOH}$ extracts of all parts of the plant had higher efficacies at the lowest concentration. The DCM extract of the stem bark showed the least activity while the $\mathrm{MeOH}$ extract of the leaves showed the highest activity compared to the others. This indicates that the $\mathrm{MeOH}$ extract of the leaves contains more 
Table 2: Bioassay data of $P$. juliflora extracts against groundnut aphid (Aphis craccivora).

\begin{tabular}{|c|c|c|c|c|c|c|c|}
\hline & \multirow{2}{*}{ Extract } & \multirow{2}{*}{ Plant part and concentration } & \multicolumn{5}{|c|}{ Mean mortality } \\
\hline & & & $12 \mathrm{~h}$ & $24 \mathrm{~h}$ & $48 \mathrm{~h}$ & $72 \mathrm{~h}$ & $96 \mathrm{~h}$ \\
\hline 1 & \multirow{10}{*}{ Hydro-distillate } & Leaves (1\%) & $3.667^{\mathrm{ij}}$ & $8.333^{\text {bcdef }}$ & $10.000^{\mathrm{a}}$ & $10.000^{\mathrm{a}}$ & $10.000^{\mathrm{a}}$ \\
\hline 2 & & Leaves(2.5\%) & $5.333^{\mathrm{hi}}$ & $8.000^{\text {cdef }}$ & $10.000^{\mathrm{a}}$ & $10.000^{\mathrm{a}}$ & $10.000^{\mathrm{a}}$ \\
\hline 3 & & Leaves(5\%) & $6.667^{\text {defgh }}$ & $9.667^{\mathrm{ab}}$ & $10.000^{\mathrm{a}}$ & $10.000^{\mathrm{a}}$ & $10.000^{\mathrm{a}}$ \\
\hline 4 & & Leaves(10\%) & $7.000^{\text {cdefgh }}$ & $9.667^{\mathrm{abcd}}$ & $10.000^{\mathrm{a}}$ & $10.000^{\mathrm{a}}$ & $10.000^{\mathrm{a}}$ \\
\hline 5 & & Leaves(15\%) & $8.333^{\text {abcdef }}$ & $10.000^{\mathrm{a}}$ & $10.000^{\mathrm{a}}$ & $10.000^{\mathrm{a}}$ & $10.000^{\mathrm{a}}$ \\
\hline 6 & & Stem bark(1\%) & $3.667^{\mathrm{ij}}$ & $9.000^{\mathrm{abcd}}$ & $10.000^{\mathrm{a}}$ & $10.000^{\mathrm{a}}$ & $10.000^{\mathrm{a}}$ \\
\hline 7 & & Stem bark(2.5\%) & $5.333^{\text {hi }}$ & $9.333^{\mathrm{ab}}$ & $10.000^{\mathrm{a}}$ & $10.000^{a}$ & $10.000^{\mathrm{a}}$ \\
\hline 8 & & Stem bark(5\%) & $7.000^{\text {cdefgh }}$ & $9.333^{\mathrm{abc}}$ & $10.000^{\mathrm{a}}$ & $10.000^{\mathrm{a}}$ & $10.000^{\mathrm{a}}$ \\
\hline 9 & & Stem bark(10\%) & $8.667^{\text {abcde }}$ & $9.333^{\mathrm{abc}}$ & $10.000^{\mathrm{a}}$ & $10.000^{\mathrm{a}}$ & $10.000^{\mathrm{a}}$ \\
\hline 10 & & Stem bark(15\%) & $9.000^{\mathrm{abcd}}$ & $10.000^{\mathrm{a}}$ & $10.000^{\mathrm{a}}$ & $10.000^{\mathrm{a}}$ & $10.000^{\mathrm{a}}$ \\
\hline 11 & \multirow{15}{*}{ Soxhlet extract } & Leaves(1\%) & $3.667^{\mathrm{ij}}$ & $8.333^{\text {bcdef }}$ & $10.000^{\mathrm{a}}$ & $10.000^{\mathrm{a}}$ & $10.000^{\mathrm{a}}$ \\
\hline 12 & & Leaves(2.5\%) & $5.333^{\text {hi }}$ & $8.667^{\text {abcde }}$ & $10.000^{\mathrm{a}}$ & $10.000^{\mathrm{a}}$ & $10.000^{\mathrm{a}}$ \\
\hline 13 & & Leaves(5\%) & $6.000^{\mathrm{fghi}}$ & $9.667^{a b}$ & $10.000^{\mathrm{a}}$ & $10.000^{\mathrm{a}}$ & $10.000^{\mathrm{a}}$ \\
\hline 14 & & Leaves(10\%) & $6.667^{\text {defgh }}$ & $9.667^{\mathrm{abcd}}$ & $10.000^{\mathrm{a}}$ & $10.000^{\mathrm{a}}$ & $10.000^{\mathrm{a}}$ \\
\hline 15 & & Leaves(15\%) & $9.333^{\mathrm{abc}}$ & $10.000^{\mathrm{a}}$ & $10.000^{\mathrm{a}}$ & $10.000^{\mathrm{a}}$ & $10.000^{\mathrm{a}}$ \\
\hline 16 & & Stem bark(1\%) & $2.333^{\mathrm{jkl}}$ & $7.000^{f}$ & $10.000^{\mathrm{a}}$ & $10.000^{\mathrm{a}}$ & $10.000^{\mathrm{a}}$ \\
\hline 17 & & Stem bark(2.5\%) & $6.333^{\text {efgh }}$ & $9.000^{\mathrm{abcd}}$ & $10.000^{\mathrm{a}}$ & $10.000^{\mathrm{a}}$ & $10.000^{\mathrm{a}}$ \\
\hline 18 & & Stem bark(5\%) & $6.667^{\text {defgh }}$ & $9.333^{\mathrm{abc}}$ & $10.000^{\mathrm{a}}$ & $10.000^{\mathrm{a}}$ & $10.000^{\mathrm{a}}$ \\
\hline 19 & & Stem bark(10\%) & $7.000^{\text {cdefgh }}$ & $9.667^{\mathrm{ab}}$ & $10.000^{\mathrm{a}}$ & $10.000^{\mathrm{a}}$ & $10.000^{\mathrm{a}}$ \\
\hline 20 & & Stem bark(15\%) & $9.667^{\mathrm{ab}}$ & $10.000^{\mathrm{a}}$ & $10.000^{\mathrm{a}}$ & $10.000^{a}$ & $10.000^{\mathrm{a}}$ \\
\hline 21 & & Seeds (1\%) & $5.667^{\mathrm{ghi}}$ & $9.333^{\mathrm{abc}}$ & $10.000^{\mathrm{a}}$ & $10.000^{\mathrm{a}}$ & $10.000^{\mathrm{a}}$ \\
\hline 22 & & Seeds(2.5\%) & $6.000^{\text {cdefgh }}$ & $9.333^{\mathrm{abc}}$ & $10.000^{\mathrm{a}}$ & $10.000^{\mathrm{a}}$ & $10.000^{\mathrm{a}}$ \\
\hline 23 & & Seeds(5\%) & $6.333^{\text {bcdefgh }}$ & $9.667^{\mathrm{ab}}$ & $10.000^{\mathrm{a}}$ & $10.000^{\mathrm{a}}$ & $10.000^{\mathrm{a}}$ \\
\hline 24 & & Seeds(10\%) & $6.667^{\text {defgh }}$ & $9.667^{\text {ef }}$ & $10.000^{\mathrm{a}}$ & $10.000^{\mathrm{a}}$ & $10.000^{\mathrm{a}}$ \\
\hline 25 & & Seeds(15\%) & $6.667^{\mathrm{fghi}}$ & $9.667^{\mathrm{abcd}}$ & $10.000^{\mathrm{a}}$ & $10.000^{\mathrm{a}}$ & $10.000^{\mathrm{a}}$ \\
\hline 26 & \multirow{15}{*}{ DCM extract } & Leaves(1\%) & $6.667^{\text {defgh }}$ & $7.667^{\text {def }}$ & $10.000^{\mathrm{a}}$ & $10.000^{\mathrm{a}}$ & $10.000^{\mathrm{a}}$ \\
\hline 27 & & Leaves(2.5\%) & $7.667^{\text {abcdefgh }}$ & $9.333^{\mathrm{abc}}$ & $10.000^{\mathrm{a}}$ & $10.000^{\mathrm{a}}$ & $10.000^{\mathrm{a}}$ \\
\hline 28 & & Leaves(5\%) & $9.333^{\mathrm{abc}}$ & $10.000^{\mathrm{a}}$ & $10.000^{\mathrm{a}}$ & $10.000^{\mathrm{a}}$ & $10.000^{\mathrm{a}}$ \\
\hline 29 & & Leaves(10\%) & $9.333^{\text {bcdefgh }}$ & $10.000^{\mathrm{abc}}$ & $10.000^{\mathrm{a}}$ & $10.000^{\mathrm{a}}$ & $10.000^{\mathrm{a}}$ \\
\hline 30 & & Leaves(15\%) & $9.667^{\mathrm{abcd}}$ & $10.000^{\mathrm{a}}$ & $10.000^{\mathrm{a}}$ & $10.000^{\mathrm{a}}$ & $10.000^{\mathrm{a}}$ \\
\hline 31 & & Stem bark(1\%) & $3.667^{\mathrm{ij}}$ & $8.333^{\text {bcdef }}$ & $10.000^{\mathrm{a}}$ & $10.000^{\mathrm{a}}$ & $10.000^{\mathrm{a}}$ \\
\hline 32 & & Stem bark(2.5\%) & $7.000^{\text {cdefgh }}$ & $9.000^{\mathrm{abcd}}$ & $10.000^{\mathrm{a}}$ & $10.000^{\mathrm{a}}$ & $10.000^{\mathrm{a}}$ \\
\hline 33 & & Stem bark(5\%) & $7.667^{\text {abcdefgh }}$ & $9.000^{\mathrm{abcd}}$ & $10.000^{\mathrm{a}}$ & $10.000^{\mathrm{a}}$ & $10.000^{\mathrm{a}}$ \\
\hline 34 & & Stem bark(10\%) & $10.000^{a}$ & $10.000^{\mathrm{a}}$ & $10.000^{\mathrm{a}}$ & $10.000^{\mathrm{a}}$ & $10.000^{\mathrm{a}}$ \\
\hline 35 & & Stem bark(10\%) & $10.000^{\mathrm{a}}$ & $10.000^{\mathrm{a}}$ & $10.000^{\mathrm{a}}$ & $10.000^{\mathrm{a}}$ & $10.000^{\mathrm{a}}$ \\
\hline 36 & & Seed(1\%) & $6.667^{\text {defgh }}$ & $10.000^{\mathrm{a}}$ & $10.000^{\mathrm{a}}$ & $10.000^{\mathrm{a}}$ & $10.000^{\mathrm{a}}$ \\
\hline 37 & & Seeds $(2.5 \%)$ & $7.000^{\text {cdefgh }}$ & $9.667^{\mathrm{ab}}$ & $10.000^{\mathrm{a}}$ & $10.000^{a}$ & $10.000^{\mathrm{a}}$ \\
\hline 38 & & Seeds (5\%) & $7.667^{\mathrm{ghi}}$ & $9.667^{\mathrm{ab}}$ & $10.000^{\mathrm{a}}$ & $10.000^{\mathrm{a}}$ & $10.000^{\mathrm{a}}$ \\
\hline 39 & & Seeds (10\%) & $9.000^{\mathrm{abcd}}$ & $10.000^{\mathrm{a}}$ & $10.000^{\mathrm{a}}$ & $10.000^{\mathrm{a}}$ & $10.000^{\mathrm{a}}$ \\
\hline 40 & & Seeds (15\%) & $9.000^{\mathrm{abcd}}$ & $10.000^{\mathrm{a}}$ & $10.000^{\mathrm{a}}$ & $10.000^{\mathrm{a}}$ & $10.000^{\mathrm{a}}$ \\
\hline 41 & \multirow{15}{*}{$\mathrm{MeOH}$ extract } & Leaves(1\%) & $9.333^{\mathrm{abc}}$ & $10.000^{\mathrm{a}}$ & $10.000^{\mathrm{a}}$ & $10.000^{\mathrm{a}}$ & $10.000^{\mathrm{a}}$ \\
\hline 42 & & Leaves(2.5\%) & $9.333^{\text {abcdefgh }}$ & $10.000^{\mathrm{a}}$ & $10.000^{\mathrm{a}}$ & $10.000^{\mathrm{a}}$ & $10.000^{\mathrm{a}}$ \\
\hline 43 & & Leaves(5\%) & $9.333^{a b c}$ & $10.000^{\mathrm{a}}$ & $10.000^{\mathrm{a}}$ & $10.000^{\mathrm{a}}$ & $10.000^{\mathrm{a}}$ \\
\hline 44 & & Leaves(10\%) & $9.333^{\mathrm{abc}}$ & $10.000^{\mathrm{a}}$ & $10.000^{\mathrm{a}}$ & $10.000^{\mathrm{a}}$ & $10.000^{\mathrm{a}}$ \\
\hline 45 & & Leaves(15\%) & $10.000^{\mathrm{a}}$ & $10.000^{\mathrm{a}}$ & $10.000^{\mathrm{a}}$ & $10.000^{\mathrm{a}}$ & $10.000^{\mathrm{a}}$ \\
\hline 46 & & Stem bark(1\%) & $8.667^{\text {abcde }}$ & $10.000^{\mathrm{a}}$ & $10.000^{\mathrm{a}}$ & $10.000^{\mathrm{a}}$ & $10.000^{\mathrm{a}}$ \\
\hline 47 & & Stem bark(2.5\%) & $9.333^{\mathrm{abc}}$ & $10.000^{\mathrm{a}}$ & $10.000^{\mathrm{a}}$ & $10.000^{\mathrm{a}}$ & $10.000^{\mathrm{a}}$ \\
\hline 48 & & Stem bark(5\%) & $9.000^{\text {abcdefg }}$ & $10.000^{\mathrm{a}}$ & $10.000^{\mathrm{a}}$ & $10.000^{\mathrm{a}}$ & $10.000^{\mathrm{a}}$ \\
\hline 49 & & Stem bark(10\%) & $9.667^{\mathrm{ab}}$ & $10.000^{\mathrm{a}}$ & $10.000^{\mathrm{a}}$ & $10.000^{\mathrm{a}}$ & $10.000^{\mathrm{a}}$ \\
\hline 50 & & Stem bark(15\%) & $9.667^{\text {abcdef }}$ & $10.000^{\mathrm{a}}$ & $10.000^{\mathrm{a}}$ & $10.000^{\mathrm{a}}$ & $10.000^{\mathrm{a}}$ \\
\hline 51 & & Seeds(1\%) & $6.000^{\text {fghi }}$ & $9.667^{\mathrm{ab}}$ & $10.000^{\mathrm{a}}$ & $10.000^{a}$ & $10.000^{\mathrm{a}}$ \\
\hline 52 & & Seeds(2.5\%) & $8.333^{\text {abcdef }}$ & $9.667^{\mathrm{ab}}$ & $10.000^{\mathrm{a}}$ & $10.000^{a}$ & $10.000^{\mathrm{a}}$ \\
\hline 53 & & Seeds(5\%) & $9.333^{\mathrm{abc}}$ & $9.667^{a b c}$ & $10.000^{\mathrm{a}}$ & $10.000^{\mathrm{a}}$ & $10.000^{\mathrm{a}}$ \\
\hline 54 & & Seeds(10\%) & $9.333^{\mathrm{abcd}}$ & $9.667^{\mathrm{abc}}$ & $10.000^{\mathrm{a}}$ & $10.000^{\mathrm{a}}$ & $10.000^{\mathrm{a}}$ \\
\hline 55 & & Seeds(15\%) & $9.667^{\mathrm{ab}}$ & $9.667^{\mathrm{ab}}$ & $10.000^{\mathrm{a}}$ & $10.000^{\mathrm{a}}$ & $10.000^{\mathrm{a}}$ \\
\hline 56 & \multirow{8}{*}{ Control } & $1 \%$ & $1.333^{\mathrm{jkl}}$ & $1.333^{\mathrm{ghi}}$ & $1.667^{\text {cde }}$ & $2.000^{\mathrm{cd}}$ & $3.000^{\mathrm{bc}}$ \\
\hline 57 & & $2.5 \%$ & $1.667^{\mathrm{jkl}}$ & $1.667^{\text {ghi }}$ & $2.000^{\mathrm{bcd}}$ & $2.333^{b c}$ & $3.000^{\mathrm{bc}}$ \\
\hline 58 & & $5 \%$ & $1.667^{\mathrm{jkl}}$ & $1.667^{\text {ghi }}$ & $2.000^{\mathrm{bcd}}$ & $2.333^{\mathrm{bc}}$ & $3.000^{\mathrm{bc}}$ \\
\hline 59 & & $10 \%$ & $2.000^{\mathrm{jkl}}$ & $2.333^{g h}$ & $2.667^{b}$ & $2.667^{b}$ & $3.000^{\mathrm{bc}}$ \\
\hline 60 & & $15 \%$ & $2.667^{\mathrm{jk}}$ & $2.667^{g}$ & $2.667^{b}$ & $2.667^{b}$ & $3.333^{b}$ \\
\hline 61 & & DMSO & $2.000^{\mathrm{jkl}}$ & $2.333^{g h}$ & $2.333^{\mathrm{bc}}$ & $2.333^{\mathrm{bc}}$ & $2.333^{\mathrm{de}}$ \\
\hline 62 & & blank & $0.667^{\mathrm{kl}}$ & $0.667^{i}$ & $1.000^{\mathrm{ef}}$ & $1.6667^{\mathrm{de}}$ & $2.333^{\mathrm{de}}$ \\
\hline 63 & & distilled water & $0.667^{\mathrm{kl}}$ & $0.667^{i}$ & $1.000^{\mathrm{ef}}$ & $1.6667^{\mathrm{de}}$ & $1.667^{\mathrm{fg}}$ \\
\hline \multicolumn{3}{|c|}{ Mean } & 5.742 & 7.227 & 5.742 & 7.227 & 7.733 \\
\hline \multicolumn{3}{|c|}{ CV(\%) } & 7.345 & 5.017 & $4 . .345$ & 3.017 & 2.211 \\
\hline \multicolumn{3}{|c|}{ LSD(0.05) } & $2.348^{* * *}$ & 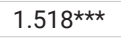 & 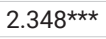 & $1.518^{\star \star \star}$ & $0.899 * * *$ \\
\hline
\end{tabular}

Citation: Zerihun M, Ele E (2020) Bioactive Compounds and Biological Assays of different Prosopis Juliflora Extracts against Groundnut Aphid. Open Journal of Chemistry 6(1): 021-029. DOI: https://dx.doi.org/10.17352/ojc.000020 

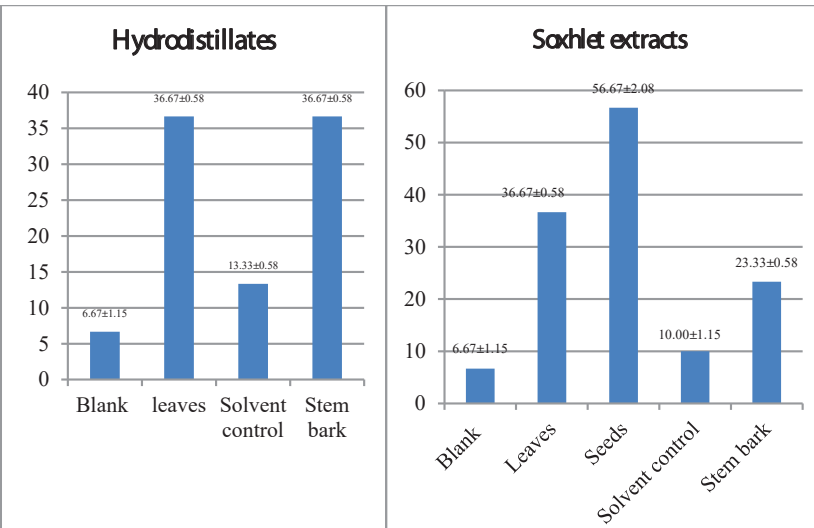
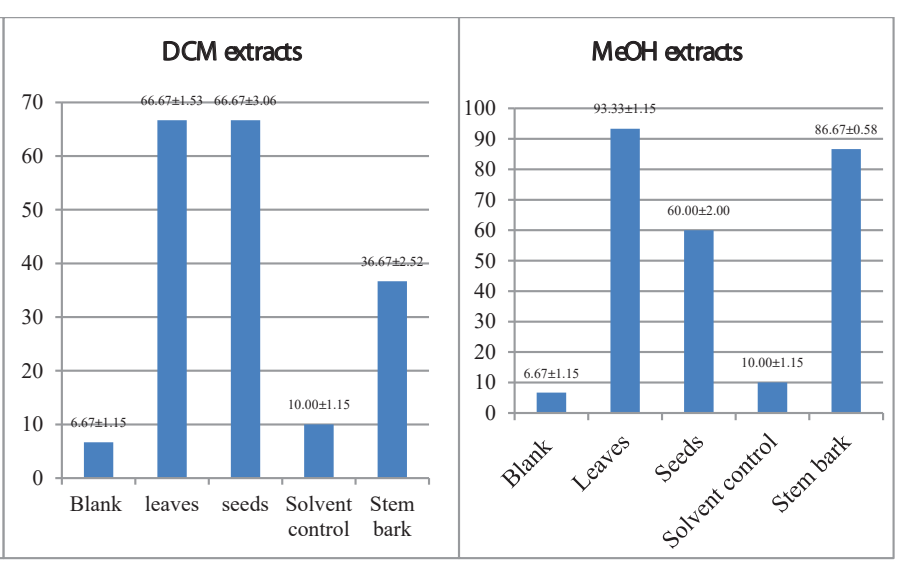

Figure 2a. Hydrodistillates extracts Figure 2b. soxhlet extracts Figure 2c. DCM extracts Figure 2d. MeOH extracts

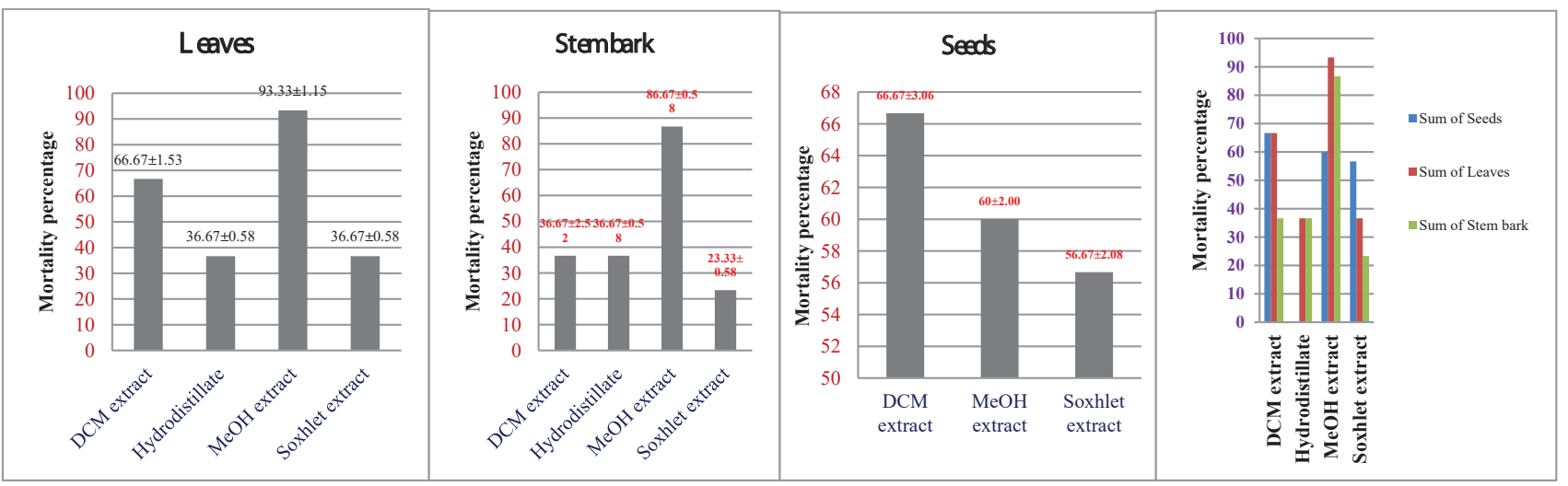

Figure 2E. leaves part Figure 2F. stem bark part Figure 2G. seeds part Figure 2H. All parts and extracts

Figure 2: Mean mortality rates of groundnut aphids due to different extracts at the lowest concentration (1\%) after $12 \mathrm{~h}$ and different parts of $P$. juliflora at the lowest concentration ( $1 \%)$ after $12 \mathrm{~h}$.

toxic compounds which may act individually or synergistically against groundnut aphids (Table 3 ).

Table 3. Efficacy of P. juliflora stem bark, seed and leaf extracts against groundnut aphids for lethal concentration $\mathrm{LC}_{50}$ and $\mathrm{LC}_{95}$ at the shortest time (12 h) and for lethal time $\mathrm{LT}_{50}$ and $\mathrm{LT}_{95}$ at the smallest concentration (1\%) after treatments.

\section{Phytochemical investigation of the leaves of $P$. juliflora}

In this work, attempts were made to isolate and characterize secondary metabolites from the leaves of $\mathrm{P}$. juliflora. Thus, two compounds were isolated by using silica gel column chromatography and characterized through different spectroscopic techniques as described below.

\section{Characterization of compound 1(4-(2-aminoethyl) phe- nol)}

Compound 1has a melting point of $160-163{ }^{\circ} \mathrm{C}$. The UV spectrum of compound $\mathbf{1}$ in $\mathrm{MeOH}$ has displayed an absorption maximum $\left(\lambda_{\max }\right)$ at $276 \mathrm{~nm}$ which is the characteristic of a phenolic nucleus ) [20]. The IR spectrum $(\mathrm{KBr})$ indicated the presence of an aromatic ring $\left(1613,1500,1229 \mathrm{~cm}^{-1}\right)$, a hydroxyl group $\left(3202 \mathrm{~cm}^{-1}\right)$ and an amine $\left(3411 \mathrm{~cm}^{-1}\right)$ group.

The ${ }^{1} \mathrm{H}$ NMR spectrum of compound 1 (Table 4) showed the doublets at $\delta 7.1$ and $6.79(\mathrm{~J}=8.0 \mathrm{~Hz})$ integrating for two protons each due to aromatic protons on a 1,4-disubstituted benzene ring. Additionally, two triplets were observed at $\delta 3.12$ and $2.87(\mathrm{~J}=8.0 \mathrm{~Hz})$ integrating for two protons each indicating the presence of two adjacent methylene groups, presumably attached to the aromatic ring at one end and to an electronwithdrawing group at the other.

The ${ }^{13} \mathrm{C}$ NMR spectrum (Table 5) of compound 1 showed a total of six carbon resonances of which four were in the aromatic region and the remaining two were in the aliphatic region(Figure 3). The DEPT-135 spectrum showed that the signals at $\delta 129.4$ and 115.3 were due to aromatic methine carbons while the signals at $\delta 40.9$ and 32.4 were due to aliphatic methylene carbons. The quaternary carbon signals have appeared at $\delta 156.4,127.0$. The ${ }^{13} \mathrm{C}$ NMR spectrum together with the ${ }^{1} \mathrm{H}$ NMR spectrum allowed the assignment of the signal at $\delta 129.4$ to the two equivalent aromatic carbons C-2 and C-6. The signal at $\delta 115.3$ can be attributed to $\mathrm{C}-3$ and $\mathrm{C}-6$ ortho to an electron-donating group at $\mathrm{C}-4$. It is thus evident that the side chain was attached to $\mathrm{C}-1$ whose carbon resonance appeared at $\delta$ 127.0.

The UV, IR and NMR data were allowed for the identification of compound 1 as 4-(2-aminoethyl) phenol. Comparison of the ${ }^{1} \mathrm{H}$ and ${ }^{13} \mathrm{C}$ NMR data of compound $\mathbf{1}$ with those reported for 
Table 3: Efficacy of $P$. juliflora stem bark, seed and leaf extracts against groundnut aphids for lethal concentration $\mathrm{LC}_{50}$ and $\mathrm{LC}_{95}$ at the shortest time (12 h) and for lethal time $\mathrm{LT}_{50}$ and $\mathrm{LT}_{95}$ at the smallest concentration (1\%) after treatments.

\begin{tabular}{|c|c|c|c|c|c|c|}
\hline \multirow{2}{*}{ Extraction methods } & \multirow{2}{*}{ Plant part } & \multirow{2}{*}{$\begin{array}{l}\text { Curve equation, } \\
\qquad \mathrm{M}=\mathrm{yC}+\mathrm{b}\end{array}$} & \multicolumn{2}{|c|}{ Lethal concentration (\%) } & \multicolumn{2}{|c|}{ Lethal time (h) } \\
\hline & & & $\mathrm{LC}_{50}$ & $\mathrm{LC}_{95}$ & $\mathrm{LT}_{50}$ & $\mathrm{LT}_{95}$ \\
\hline \multirow{3}{*}{$\begin{array}{c}\text { Hydro- } \\
\text { distiliation }\end{array}$} & Seeds & - & - & - & - & - \\
\hline & Stem bark & $M=14.000 C+11.334$ & 2.76 & 5.98 & 1.46 & 4.76 \\
\hline & Leaves & $M=10.999 C+18.004$ & 2.91 & 7.00 & 1.63 & 4.77 \\
\hline \multirow{3}{*}{ Soxhlet extraction } & Seeds & $M=2.667 C+52.000$ & $\mathrm{HS}$ & 16.12 & $\mathrm{HS}$ & 4.54 \\
\hline & Stem bark & $M=15.335 C+2.660$ & 3.09 & 6.02 & 2.44 & 4.89 \\
\hline & Leaves & $M=12.666 C+11.336$ & 3.05 & 6.61 & 1.63 & 4.77 \\
\hline \multirow{3}{*}{ DCM extraction } & Seeds & $M=6.666 C+52.004$ & HS & 6.45 & $\mathrm{HS}$ & 4.25 \\
\hline & Stem bark & $M=15.666 C+14.004$ & 2.30 & 5.17 & 1.63 & 4.77 \\
\hline & Leaves & $M=7.666 C+54.670$ & HS & 5.26 & HS & 4.70 \\
\hline \multirow{3}{*}{$\mathrm{MeOH}$ extraction } & Seeds & $M=8.334 C+51.996$ & HS & 5.16 & HS & 4.44 \\
\hline & Stem bark & $M=2.334 C+83.998$ & HS & 4.71 & $\mathrm{HS}$ & 3.12 \\
\hline & Leaves & $M=1.334 C+89.328$ & HS & 4.25 & HS & 1.25 \\
\hline
\end{tabular}

Table 4: Comparison of ${ }^{1} \mathrm{H}$ NMR data of compound 1 with With literature values $\left(\mathrm{inD}_{2} \mathrm{O}\right)$.

\begin{tabular}{|c|c|c|}
\hline Proton & $\begin{array}{c}\mathbf{1} \\
\left(\boldsymbol{(}_{\text {pom }}\right)\end{array}$ & $\begin{array}{c}\text { Literature }[\mathbf{2 1}, \mathbf{2 2}] \\
\left(\boldsymbol{(}_{\text {pom }}\right)\end{array}$ \\
\hline 2,6 & $7.12\left(2 \mathrm{H}, d, J=8.0 \mathrm{~Hz}, \mathrm{H}-2\right.$ and $\left.2^{\prime}\right)$ & 7.24 \\
\hline 3,5 & $6.79\left(2 \mathrm{H}, d, J=8.0 \mathrm{~Hz}, \mathrm{H}-3\right.$ and $\left.3^{\prime}\right)$ & 6.90 \\
\hline $1^{\prime}$ & $2.87(2 \mathrm{H}, t, J=8.0 \mathrm{~Hz}, \mathrm{H}-6)$ & $2.94(2 \mathrm{H})$ \\
\hline $2^{\prime}$ & $3.12(2 \mathrm{H}, t, J=8.0 \mathrm{~Hz}, \mathrm{H}-5)$ & $3.16(2 \mathrm{H})$ \\
\hline
\end{tabular}

Table 5: Comparison of ${ }^{13} \mathrm{C}$ NMR data of compound 1 with literature (Sato., et al. 1970), (Samsonova., et al. 2004) values (in DMSO-d $\mathrm{d}_{6}$ ).

\begin{tabular}{|c|c|c|}
\hline Carbon & $\mathbf{1}$ & $\begin{array}{c}\text { Literature }[\mathbf{2 1 , 2 2 ]} \\
\left(\mathbf{(}_{\mathbf{p o m}}\right)\end{array}$ \\
\hline 1 & 127.0 & 127.2 \\
\hline 2,5 & 129.4 & 129.4 \\
\hline 3,6 & 115.3 & 115.4 \\
\hline 4 & 156.4 & 156.2 \\
\hline $1^{\prime}$ & 32.4 & 30.6 \\
\hline $2^{\prime}$ & 40.9 & 38.7 \\
\hline
\end{tabular}

4-(2-aminoethyl) phenol in the literature $[21,22]$ revealed that the two compounds are identical(Figure 3).

\section{Characterization of compound 2 (3-0-methyl-chiro-ino- sitol)}

Compound 2 has a melting point of $158-161^{\circ} \mathrm{C}$. The UV spectrum of compound 2 in $\mathrm{MeOH}$ did not show any absorption band above $200 \mathrm{~nm}$. This indicated that there are no chromophores and that it might be a saturated hydrocarbon. The IR spectrum $(\mathrm{KBr})$ indicated the presence of hydroxyl groups $\left(3306 \mathrm{~cm}^{-1}\right.$ ). An aliphatic $\mathrm{C}-\mathrm{H}$ stretching band has appeared at $2913 \mathrm{~cm}^{-1}$ and the $\mathrm{O}-\mathrm{C}$ stretching band was observed at $1500 \mathrm{~cm}^{-1}$ while bands between 1246 and $1447 \mathrm{~cm}^{-1}$ were due to bending vibration of the $\mathrm{O}-\mathrm{C}$ group.

The ${ }^{1} \mathrm{H}$ NMR spectrum of compound 2 (in $\mathrm{D}_{2} \mathrm{O}$ ) showed nine proton resonances between $\delta 3.25$ and 3.88 which indicates all the carbon atoms to which the protons are attached are oxygenated. The proton resonances at $\delta 3.88,3.70,3.67,3.62$, 3.55 , and 3.24 were integrated to one proton each and due to methine protons while the signal at $\delta 3.46$ was integrated for three protons and due to a methoxy group. The ${ }^{1} \mathrm{H}$ NMR spectrum was recorded in DMSO- $\mathrm{d}_{6}$ (Table 6) showed five additional proton resonances at $\delta 4.73$ (d), 4.69 (d), 4.54 (d), 4.49 (d), 4.35 (d) which integrated for one proton each. These signals were disappeared upon the addition of few drops of $\mathrm{D}_{2} \mathrm{O}$ and therefore revealed the presence of five hydroxyl groups.

The ${ }^{13} \mathrm{C}$ NMR spectrum of compound 2 showed a total of seven carbon resonances between $\delta 60.1$ and 84.2. The DEPT135 spectrum revealed that the signal at $\delta 60.1$ was due to a methoxy group while the remaining carbon resonances were attributable to methine carbons.

It was evident from the spectroscopic data generated for compound 2 to be a polyhydroxylated hydrocarbon. The ${ }^{1} \mathrm{H},{ }^{13} \mathrm{C}$ and DEPT-135 NMR spectra together with the literature data indicated that the proposed compound 2 was identified to be 3-O-chiro-inositol (Figure 4). The HH COSY and HSQC spectra established the correlation which was in agreement with the proposed structure. Comparison of the ${ }^{1} \mathrm{H}$ and $2 \mathrm{D}$ NMR data of compound 2 with those reported in the literature [23,24] for 3-O-methyl-chiro-inositol revealed a very close resemblance.

\section{Conclusion}

The present study was conducted to evaluate the insecticidal activities of different extracts of P. juliflora against groundnut aphids (Aphiscraccivora). All extracts of P. juliflora showed a high percentage of mortality at $1 \%$ concentration in $24 \mathrm{~h}$ against groundnut aphids. Different extracts of P. juliflora showed a significant difference in the insecticidal activity at a $0.001 \%$ level of confidence. Thus, P. juliflora has the potential to be used as a bio-insecticide. As part of this research, the phytochemical

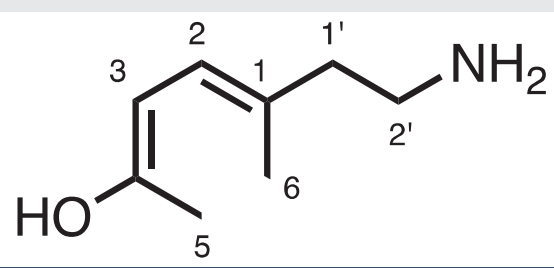

Figure 3: Structure of compound 1(4-(2-aminoethyl) phenol). 
Table 6: ${ }^{1} \mathrm{H}$ NMR $\left(400.13 \mathrm{MHz}\right.$, in $\left.\mathrm{D}_{2} \mathrm{O}\right)$ and ${ }^{13} \mathrm{C}$ NMR $\left(100.6 \mathrm{MHz}\right.$, DMSO- $\left.\mathrm{d}_{6}\right)$ data of compound 2 .

\begin{tabular}{|c|c|c|c|}
\hline Proton & $\mathbf{2}$ & Literature [23,24] & Carbon (2) \\
\hline 1 & 3.70 & 3.89 & 72.4 \\
\hline 2 & 3.62 & 3.62 & 71.3 \\
\hline 3 & 3.24 & 3.25 & 84.2 \\
\hline 4 & 3.46 & 3.35 & 73.0 \\
\hline 5 & 3.67 & 3.73 & 70.5 \\
\hline 6 & 3.88 & 3.91 & 72.9 \\
\hline 7 & 3.55 & 3.58 & 60.1 \\
\hline & & & \\
\hline & & & \\
\hline
\end{tabular}

Figure 4: Structure of Compound 2 (3-0-methyl-chiro-inositol).

analysis was conducted on the $\mathrm{MeOH}$ extract of the leaves of P. juliflora and two compounds, namely, 4-(2-aminoethyl) phenol (1), and 3-O-methyl-chiro-inositol (2) were isolated and characterized through different spectroscopic techniques. A review of the chemical literatures showed that P. juliflora is a rich source of flavonoids, alkaloids and saponins. A more rigorous bioactivity-guided phytochemical work on P. juliflora might lead to the isolation and characterization of novel secondary metabolites with insecticidal properties.

\section{Acknowledgment}

The authors would like to acknowledge the Department of Chemistry, AAU, for providing the laboratory facilities and the Ethiopian Institute of Agricultural Research (EIAR) for giving Mr. Mulate full sponsorship to attend the MSc program. The authors thank the Ethiopian institution of agricultural research especially the food science and nutrition directorate for providing all necessary technical and financial support. Thanks go to Melkassa agriculture research food science and nutrition researcher staff for their technical assistance and laboratory facility. The abstract of this manuscript has been released as a pre-print at https://academicjournals.org/journal/IJBMBR/ article-in-press abstract/extraction__ isolation _ and characterization_of_pure_compounds_from_prosopis_ juliflora_and_determination_of_the_effects_of_the_ compounds_on_groundnut_aphids

\section{References}

1. Mann J (1995) Secondary Metabolism, Oxford University Press, New York Link: https://bit.ly/2lkiaNj

2. Mann J (1994) Chemical Aspects of Biosynthesis, Oxford University Press.

3. Burkart A (1976) A monograph of the genus Prosopis (Leguminosae subfam. Mimosoideae). Journal of the Arnold Arboretum 57: 450-525. Link: https://bit.ly/3ncqAVu
4. Felker $P$, Clark PR, Laag A, Pratt $P$ (1981) Salinity tolerance of the tree legumesgrown in sand culture on nitrogen-free media. Plant Soil 61: 311-317. Link: https://bit.ly/3nhbYUV

5. Van Klinken R, Campbell S, Heard T, McKenzie J, March N (2001) The biology of Australian weeds. Prosopis L. species. Plant Protection Quarterly 16: 2-20. Link: https://bit.ly/3lsOhsj

6. Abdulahi M, Regasa T (2017) Prosopis juliflora L: distribution, impacts and available control methods in ETHIOPIA. Tropical and Subtropical Agroecosystems 20: 75-89. Link: https://bit.ly/38x86uZ

7. Birhane E, Treydte AC, Eshete A, Solomon N, Hailemariam M (2017) Carbon stocks of communal grazing lands invaded by Prosopis juliflora. Journal of Arid Environments 141: 60-67. Link: https://bit.ly/2GT8d8G

8. Sen D, Chawan D (1970) Ecology of Desert Plants and Observations on Their Seedlings: III: The Influence of Aqueous Extracts of Prosopis juliflora DC. on Euphorbia caducifolia Haines. Vegetatio 21: 277-298. Link: https://bit.ly/2GSPHNG

9. Al-Rawahy SH, Al-Dhafri K, Al-Bahlany S (2003) Germination, growth and drought resistance of native and alien plant species of the genus Prosopis in the sultanate of Oman. Asian J Plant Sci 2: 1020-1023. Link: https://bit.ly/2JVDMzL

10. Wise R, Van Wilgen B, Le Maitre D (2012) Costs, benefits and management options for an invasive alien tree species: the case of mesquite in the Northern Cape, South Africa. Journal of Arid Environments 84: 80-90. Link: https://bit.ly/32C6Frq

11. Silva AM, Silva AR, Pinheiro AM, Freitas SR, Silva VD, et al. (2007) Alkaloids from Prosopis juliflora leaves induce glial activation, cytotoxicity and stimulate NO production. Toxicon 49: 601-614. Link: https://bit.ly/3pnRiwk

12. Singh S, Swapnil V (2011) Antibacterial properties of alkaloid rich fractions obtained from various parts of Prosopis juliflora. Int J Pharm Sci Res 2: 114120. Link: https://bit.ly/2UjlGsp

13. Satish S, Raveesha V, Janardhana G (1999) Antibacterial activity of plant extracts on phytopathogenic Xanthomonas campestris pathovars. Letters Applied Microbiology 28: 145-147. Link: https://bit.ly/36qHIjP

14. Al-Shakh-Hamed W, Al-Jammas M (1999) The antimicrobial activity of alkaloidal fraction of Prosopis juliflora. Iraqi Journal Veterinary Science 12: 281-287.

15. Kupchan SM, Tsou G (1973) Structure and partial synthesis of fabacein Journal of Organic Chemistry 38: 1055-1056

16. VanWagenen BC, Larsen R, Cardellina JH, Randazzo D, Lidert ZC, et al. (1993) Ulosantoin, a potent insecticide from the sponge Ulosa ruetzleri $\mathrm{J}$ Org Chem 58: 335-337. Link: https://bit.ly/32C6Tig

17. Jørgensen $K$, Rasmussen AV, Morant $M$, Nielsen $A H$, Bjarnholt $N$, et al (2005) Metabolon formation and metabolic channeling in the biosynthesis of plant natural products. Current Opinion in Plant Biology 8: 280-291. Link: https://bit.ly/38ATTx5

18. Ghosh A, Chowdhury N, Chandra G (2012) Plant extracts as potential mosquito larvicides. Indian J Med Res 5: 581-598. Link: https://bit.ly/3eYFQ5B

19. Ashford J, Sowden R (1970) Multi-variate probit analysis. Biometrics 26: 535546. Link: https://bit.ly/3njYXKy

20. Sayed H, Mohamed M, Farag S, Mohamed G, Ebel R, et al. (2006) Phenolics of cyperus alopecuroides rottb. inflorescences and their biological activities. Bulletin of Pharmaceutical Sciences 29: 9-32. Link: https://bit.ly/3lpBRS4

21. Sato H, Sakamura S, Obata Y (1970) The isolation and characterization of $\mathrm{N}$-methyltyramine, tyramine and hordenine from sawa millet seeds. Agricultural and Biological Chemistry 34: 1254-1255. Link: https://bit.ly/38AID4N

Citation: Zerihun M, Ele E (2020) Bioactive Compounds and Biological Assays of different Prosopis Juliflora Extracts against Groundnut Aphid. Open Journal of Chemistry 6(1): 021-029. DOI: https://dx.doi.org/10.17352/ojc.000020 
22. Samsonova JV, Uskova NA, Andresyuk AN, Franek M, ElliottC T (2004) Biacore biosensor immunoassay for 4-nonylphenols: assay optimization and applicability for shellfish analysis. Chemosphere 57: 975-985. Link: https://bit.ly/2UmdpFm

23. DellaGreca M, Fiorentino A, IzzoA, NapoliF, Purcaro R, et al. (2007) Phytotoxicity of secondary metabolites from Aptenia cordifolia. Chem Biodivers 4: 118-128. Link: https://bit.ly/38AUleT
24. Abraham RJ, ByrneJ J, Griffiths L, Koniotou R (2005) $1 \mathrm{H}$ chemical shifts in NMR: Part 22-Prediction of the $1 \mathrm{H}$ chemical shifts of alcohols, diols and inositols in solution, a conformational and solvation investigation. Magn Reson Chem 43: 611-624. Link: https://bit.ly/3kmHnne
Discover a bigger Impact and Visibility of your article publication with

Peertechz Publications

\section{Highlights}

* Signatory publisher of ORCID

* Signatory Publisher of DORA (San Francisco Declaration on Research Assessment)

* Articles archived in worlds' renowned service providers such as Portico, CNKI, AGRIS, TDNet, Base (Bielefeld University Library), CrossRef, Scilit, J-Gate etc.

* Journals indexed in ICMJE, SHERPA/ROMEO, Google Scholar etc.

* OAI-PMH (Open Archives Initiative Protocol for Metadata Harvesting)

* Dedicated Editorial Board for every journal

* Accurate and rapid peer-review process

* Increased citations of published articles through promotions

* Reduced timeline for article publication

Submit your articles and experience a new surge in publication services (https://www.peertechz.com/submission).

Peertechz journals wishes everlasting success in your every endeavours.

Copyright: @ 2020 Zerihun M, et al. This is an open-access article distributed under the terms of the Creative Commons Attribution License, which permits unrestricted use, distribution, and reproduction in any medium, provided the original author and source are credited.

Citation: Zerihun M, Ele E (2020) Bioactive Compounds and Biological Assays of different Prosopis Juliflora Extracts against Groundnut Aphid. Open Journal of Chemistry 6(1): 021-029. DOI: https://dx.doi.org/10.17352/ojc.000020 